\title{
Rainbow matchings in properly edge colored graphs
}

\author{
Guanghui Wang \\ School of Mathematics \\ Shandong University \\ Jinan, Shandong, 250100, P.R. China \\ ghwang@sdu.edu.cn
}

Submitted: Mar 14, 2011; Accepted: Jul 20, 2011; Published: Aug 5, 2011

Mathematics Subject Classifications: 05C15, 05C70

\begin{abstract}
Let $G$ be a properly edge colored graph. A rainbow matching of $G$ is a matching in which no two edges have the same color. Let $\delta$ denote the minimum degree of $G$. We show that if $|V(G)| \geq \frac{8 \delta}{5}$, then $G$ has a rainbow matching of size at least $\left\lfloor\frac{3 \delta}{5}\right\rfloor$. We also prove that if $G$ is a properly colored triangle-free graph, then $G$ has a rainbow matching of size at least $\left\lfloor\frac{2 \delta}{3}\right\rfloor$.
\end{abstract}

Keywords: rainbow matchings, properly colored graphs, triangle-free graphs

\section{Introduction and notation}

We use [3] for terminology and notations not defined here and consider simple undirected graphs only. Let $G=(V, E)$ be a graph. A proper edge-coloring of $G$ is a function $c: E \rightarrow \mathbb{N}$ ( $\mathbb{N}$ is the set of nonnegative integers) such that any two adjacent edges have distinct colors. If $G$ is assigned such a coloring $c$, then we say that $G$ is a properly edgecolored graph, or simply a properly colored graph. Let $c(e)$ denote the color of the edge $e \in E$. For a subgraph $H$ of $G$, let $c(H)=\{c(e): e \in E(H)\}$. A subgraph $H$ of $G$ is called rainbow if its edges have distinct colors. Recently rainbow subgraphs have received much attention, see the survey paper [8]. Here we are interested in rainbow matchings. The study of rainbow matchings began with the following conjectures.

Conjecture 1 (Ryser [5]) Every Latin square of odd order has a Latin transversal.

Conjecture 2 (Brualdi-Stein [9, 11]) Every latin square of order $n$ has a partial Latin transversal of size at least $n-1$.

An equivalent statement is that every proper $n$-edge-coloring of the complete bipartite graph $K_{n, n}$ contains a rainbow matching of size $n-1$; Moreover, if $n$ is odd, there exists 
a rainbow perfect matching. Hatami and Shor [7] proved that there is always a partial Latin transversal (rainbow matching) of size at least $n-O\left(\log ^{2} n\right)$.

Another topic related to rainbow matchings is orthogonal matchings of graphs. Let $G$ be a graph on $n$ vertices which is an edge disjoint union of $m k$-factors (i.e. $k$ regular spanning subgraphs). We ask if there is a matching $M$ of $m$ edges with exactly one edge from each $k$-factor? Such a matching is called orthogonal because of applications in design theory. A matching $M$ is suborthogonal if there is at most one edge from each $k$-factor. Alspach [1] posed the above problem in the case $k=2$. Stong [10] proved that if $n \geq 3 m-2$, then there is a such orthogonal matching. For $k=3$, the answer is yes, see [2]. In the same paper, Anstee and Caccetta proved the following theorem when $k=1$.

Theorem 2 [2] Let $G$ be an m-regular graph on $n$ vertices. Then for any decomposition of $E(G)$ into $m$ 1-factors $F_{1}, F_{2}, \ldots, F_{m}$, there is a matching $M$ of $p$ edges, at most one edge from each 1-factor, with

$$
p>\min \left\{\frac{n}{2}-\frac{3}{2}\left(\frac{n}{2}\right)^{\frac{2}{3}}, m-\frac{3}{2} m^{\frac{2}{3}}\right\}
$$

In any decomposition of $E(G)$ into $m k$-factors, we can construct an edge-colored graph by giving each $k$-factor a color. Then a rainbow matching of $G$ corresponds to a suborthogonal matching of $G$. In particular, when $k=1$, the edge-colored graph obtained above is properly colored. So we can pose a more general problem: Let $G$ be a properly colored graph of minimum degree $\delta(G)$. Is there a rainbow matching of size $\delta(G)$ ? Unfortunately, the answer is negative: Let $C_{4}^{2}$ denote a properly 2-edge-colored cycle with four vertices and $K_{4}^{3}$ be a properly 3-edge-colored complete graph with four vertices. Let $K_{4}^{3}-e$ denote the graph obtained from $K_{4}^{3}$ by deleting an edge. Then there is no rainbow matchings of size two in $C_{4}^{2}, K_{4}^{3}$, or $K_{4}^{3}-e$. Moreover, if $G$ is a properly colored complete graph, then $G$ has no rainbow matching of size more than $\left\lceil\frac{\delta(G)}{2}\right\rceil$. In addition, the following theorem was shown in [6].

Theorem $3[6]$ Let $G$ be a properly colored graph, $G \neq K_{4}$, and $|V(G)| \neq \delta(G)+2$. Then $G$ contains a rainbow matching of size $\left\lceil\frac{\delta(G)}{2}\right\rceil$.

However, we believe that if the order of a properly colored graph $G$ is much larger than its minimum degree $\delta(G)$, there should be a rainbow matching of size $\delta(G)$. So we propose the following problem.

Problem 4 Is there a function $f(n)$ such that for each properly colored graph $G$ with $|V(G)| \geq f(\delta(G)), G$ must contain a rainbow matching of size $\delta(G)$ ?

Since when $n$ is even, there exists an $n \times n$ Latin square that has no Latin transversal (perfect rainbow matching) (see $[4,11]$ ), if the function $f(n)$ exists, $f(n)$ should be greater than $2 n$. Motivated by this problem, we prove the following results. 
Theorem 5 Let $G$ be a properly colored graph and $|V(G)| \geq \frac{8 \delta(G)}{5}$. Then $G$ has a rainbow matching of size at least $\left\lfloor\frac{3 \delta(G)}{5}\right\rfloor$.

Theorem 6 Let $G$ be a properly colored triangle-free graph. Then $G$ has a rainbow matching of size at least $\left\lfloor\frac{2 \delta(G)}{3}\right\rfloor$.

\section{Proof of Theorem 5}

For simplicity, let $\delta=\delta(G)$. If $\delta \leq 3$, it is easy to check that our theorem holds. If $4 \leq \delta \leq 9$, by Theorem $3, G$ contains a rainbow matching of size $\left\lceil\frac{\delta}{2}\right\rceil$. Since $\left\lceil\frac{\delta}{2}\right\rceil \geq\left\lfloor\frac{3 \delta}{5}\right\rfloor$, when $4 \leq \delta \leq 9$, our conclusion holds too. So now we assume that $\delta \geq 10$. We will prove it by contradiction. Suppose our conclusion is not true. We choose a maximum rainbow matching $M$. Let $t=|E(M)|$. Then $t \leq\left\lfloor\frac{3 \delta}{5}\right\rfloor-1$. Suppose that $E(M)=\left\{e_{1}, e_{2}, \ldots, e_{t}\right\}$ and $e_{i}=x_{i} y_{i}$. Moreover, without loss of generality, we assume that $c\left(e_{i}\right)=i$, for $1 \leq i \leq t$. Put $V_{1}=V-V(M)$. We call a color a new color if it is not in $c(M)$ and call an edge $u v$ special if $v \in V(M), u \in V_{1}$ and $c(u v)$ is a new color. For $v \in V(M)$, let $d_{s}(v)$ denote the number of the special edges incident with $v$. Let $V_{2}$ denote the vertices $v \in V(M)$ with $d_{s}(v) \geq 4$. We have the following claim.

Claim 1. For each edge $x_{i} y_{i} \in E(M)$, if $d_{s}\left(x_{i}\right)+d_{s}\left(y_{i}\right) \geq 5$, then either $d_{s}\left(x_{i}\right)=0$ or $d_{s}\left(y_{i}\right)=0$.

Proof. Otherwise, it holds that $d_{s}\left(x_{i}\right)+d_{s}\left(y_{i}\right) \geq 5$ and $d_{s}\left(x_{i}\right), d_{s}\left(y_{i}\right) \geq 1$. Then one of $d_{s}\left(x_{i}\right), d_{s}\left(y_{i}\right)$ is at least 3 . Suppose that $d_{s}\left(x_{i}\right) \geq 3$. Since $d_{s}\left(y_{i}\right) \geq 1$, we choose a special edge $y_{i} u$. As $d_{s}\left(x_{i}\right) \geq 3$, we can also choose a special edge $x_{i} w$ such that $c\left(x_{i} w\right) \neq c\left(y_{i} u\right)$ and $w \neq u$. Now $M \cup\left\{x_{i} w, y_{i} u\right\} \backslash x_{i} y_{i}$ is a rainbow matching of size $t+1$, a contradiction.

Claim 2. $\left|V_{2}\right| \geq\left\lceil\frac{2 \delta}{5}\right\rceil$.

Proof. Let $x \in V_{1}$. If there is an edge $x y$ such that $c(x y) \notin c(M)$, then $y \in V(M)$. Otherwise, there is a rainbow matching $M \cup x y$ of size $t+1$, which is a contradiction. Let $E_{s}$ denote the set formed by all special edges. Since each vertex in $V_{1}$ has degree at least $\delta,\left|E_{s}\right| \geq(\delta-t)\left|V_{1}\right| \geq\left(\left\lceil\frac{2 \delta}{5}\right\rceil+1\right)\left|V_{1}\right|$. By Claim 1, for each edge $x_{i} y_{i} \in E(M)$, if $d_{s}\left(x_{i}\right)+d_{s}\left(y_{i}\right) \geq 5$, then $d_{s}\left(x_{i}\right)=0$ or $d_{s}\left(y_{i}\right)=0$, so $d_{s}\left(x_{i}\right)+d_{s}\left(y_{i}\right) \leq\left|V_{1}\right|$; If $d_{s}\left(x_{i}\right)+$ $d_{s}\left(y_{i}\right) \leq 4$, recall that $\left|V_{1}\right|=|V(G)|-|V(M)| \geq \frac{8 \delta}{5}-2\left(\left\lfloor\frac{3 \delta}{5}\right\rfloor-1\right) \geq \frac{2 \delta}{5}+2 \geq 5$, thus $d_{s}\left(x_{i}\right)+d_{s}\left(y_{i}\right) \leq\left|V_{1}\right|$. Hence $\left|E_{s}\right| \leq\left|V_{2}\right|\left|V_{1}\right|+4\left(|E(M)|-\left|V_{2}\right|\right)$. This implies $\left(\left\lceil\frac{2 \delta}{5}\right\rceil+1\right)\left|V_{1}\right| \leq\left|V_{2}\right|\left|V_{1}\right|+4\left(|E(M)|-\left|V_{2}\right|\right)$. Hence

$$
\begin{aligned}
\left|V_{2}\right| \geq \frac{\left(\left\lceil\frac{2 \delta}{5}\right\rceil+1\right)\left|V_{1}\right|-4|E(M)|}{\left|V_{1}\right|-4} & \geq \frac{\left(\left\lceil\frac{2 \delta}{5}\right\rceil+1\right)\left|V_{1}\right|-4\left(\left\lfloor\frac{3 \delta}{5}\right\rfloor-1\right)}{\left|V_{1}\right|-4} \\
& =\left\lceil\frac{2 \delta}{5}\right\rceil+1-\frac{4\left\lfloor\frac{3 \delta}{5}\right\rfloor-4\left\lceil\frac{2 \delta}{5}\right\rceil-8}{\left|V_{1}\right|-4} .
\end{aligned}
$$


Since $\left|V_{1}\right| \geq \frac{2 \delta}{5}+2,\left|V_{2}\right| \geq\left\lceil\frac{2 \delta}{5}\right\rceil$.

By Claim 1, there cannot be an edge in $M$ such that both end vertices of this edge are in $V_{2}$. Then, without loss of generality, we assume that $V_{2}=\left\{x_{1}, x_{2}, \ldots, x_{p}\right\}$, where $p=\left|V_{2}\right| \geq\left\lceil\frac{2 k}{5}\right\rceil$. Let $G^{\prime}$ denote the subgraph induced by $\left\{y_{1}, y_{2}, \ldots, y_{p}\right\}$.

Claim 3. No color in $c\left(E\left(G^{\prime}\right)\right)$ is a new color.

Proof. Suppose, to the contrary, there exists an edge, say $y_{1} y_{2}$ such that $c\left(y_{1} y_{2}\right)$ is a new color. Then we can find two independent edges $x_{1} w_{1}$ and $x_{2} w_{2}$ such that $w_{1}, w_{2} \in V_{1}$, $c\left(x_{1} w_{1}\right), c\left(x_{2} w_{2}\right) \notin c(M) \cup\left\{c\left(y_{1} y_{2}\right)\right\}$ and $c\left(x_{1} w_{1}\right) \neq c\left(x_{2} w_{2}\right)$. We can do this, since each vertex in $V_{2}$ is incident with four special edges. Now we obtain a rainbow matching $M \cup\left\{x_{1} w_{1}, x_{2} w_{2}, y_{1} y_{2}\right\} \backslash\left\{x_{1} y_{1}, x_{2} y_{2}\right\}$ of size $t+1$, which is a contradiction.

Claim 4. $c\left(E\left(G^{\prime}\right)\right) \cap\{1,2, \ldots, p\}=\emptyset$.

Proof. Otherwise, there is an edge, say $y_{1} y_{2}$ such that $c\left(y_{1} y_{2}\right) \in\{1, \ldots, p\}$. We assume that $c\left(y_{1} y_{2}\right)=j$. We know that $G$ is properly colored, so $j \neq 1,2$. For convenience, assume that $j=3$. We will show the following fact.

Fact. There exists a rainbow matching formed by three special edges $\left\{x_{1} u_{1}, x_{2} u_{2}, x_{3} u_{3}\right\}$.

Proof of the Fact. We prove it by contradiction. We choose three special edges incident with $x_{1}, x_{2}, x_{3}$ to form a matching $M_{1}$ such that $\left|c\left(M_{1}\right)\right|$ is as large as possible. Since each $x_{i}$ is incident with four special edges and by our assumption, we can assume that $\left|c\left(M_{1}\right)\right|=2$. Without loss of generality, assume that $M_{1}=\left\{x_{1} u, x_{2} v, x_{3} w\right\}$ and $c\left(x_{1} u\right)=$ $a_{1}, c\left(x_{2} v\right)=a_{2}, c\left(x_{3} w\right)=a_{1}$. As $x_{3}$ is incident with four special edges, there are two special edges $x_{3} v_{1}, x_{3} v_{2}$ such that $v_{1}, v_{2} \in V_{1}$ and $c\left(x_{3} v_{1}\right), c\left(x_{3} v_{2}\right) \notin c(M) \cup\left\{a_{1}, a_{2}\right\}$. We claim that $\left\{v_{1}, v_{2}\right\}=\{u, v\}$, otherwise we will get a rainbow matching satisfying our condition. Now we assume that $c\left(x_{3} u\right)=a_{3}, c\left(x_{3} v\right)=a_{4}$. Similarly, we assume that $c\left(x_{1} v\right)=b_{1}$ and $c\left(x_{1} w\right)=b_{2}$, where $b_{1}, b_{2} \notin c(M) \cup\left\{a_{1}, a_{2}\right\}$. Then $b_{2}=a_{3}$, otherwise $\left\{x_{1} w, x_{3} u, x_{2} v\right\}$ forms a rainbow matching, which is a contradiction. Moreover, $b_{1} \neq a_{4}$, since $G$ is properly colored.

Now consider the vertex $x_{2}$. Since $x_{2}$ is incident with four special edges, there is an edge, say $x_{2} z$ such that $c\left(x_{2} z\right) \notin c(M) \cup\left\{a_{2}\right\}$ and $z \notin\{u, v, w\}$. Then $c\left(x_{2} z\right)=a_{3}$, otherwise either $\left\{x_{2} z, x_{1} v, x_{3} u\right\}$ or $\left\{x_{2} z, x_{1} w, x_{3} v\right\}$ would be a rainbow matching, and we are done. Hence $\left\{x_{2} z, x_{1} v, x_{3} w\right\}$ is a rainbow matching with colors $\left\{a_{3}, a_{1}, b_{1}\right\}$, which is a contradiction. This completes the proof of the fact.

By the above fact, $M \cup\left\{x_{1} u_{1}, x_{2} u_{2}, x_{3} u_{3}, y_{1} y_{2}\right\} \backslash\left\{e_{1}, e_{2}, e_{3}\right\}$ is a rainbow matching of size $t+1$. This contradiction completes the proof of Claim 4 .

Claim 5. If there is an edge $y_{j} u$, where $y_{j} \in V\left(G^{\prime}\right)$ and $u \in V_{1}$, then $c\left(y_{j} u\right) \in c(M)$ and $c\left(y_{j} u\right) \cap\{1,2, \ldots, p\}=\emptyset$. 
Proof. Otherwise, suppose that $c\left(y_{j} u\right)$ is a new color. Then $d_{s}\left(y_{j}\right) \geq 1$. Since $d_{s}\left(x_{j}\right) \geq$ $4, d_{s}\left(x_{j}\right)+d_{s}\left(y_{j}\right) \geq 5$, which contradicts with Claim 1. So $c\left(y_{j} u\right) \in c(M)$. Suppose $c\left(y_{j} u\right)=k$, where $1 \leq k \leq p$. Since $G$ is properly colored, $k \neq j$. Since $x_{j}, x_{k} \in V_{2}$, we can find a special edge $x_{j} w_{1}$ such that $w_{1} \neq u$. Next, there is a special edge $x_{k} w_{2}$ such that $w_{2} \notin\left\{u, w_{1}\right\}$ and $c\left(x_{k} w_{2}\right) \neq c\left(x_{j} w_{1}\right)$. Hence we have a rainbow matching $M \cup\left\{x_{j} w_{1}, x_{k} w_{2}, y_{j} u\right\} \backslash\left\{x_{j} y_{j}, x_{k} y_{k}\right\}$, which is a contradiction. Thus Claim 5 holds.

Now consider a vertex $y_{j}$, where $1 \leq j \leq p$. By Claims 3,4, and 5, we know that if $y_{j}$ has a neighbor $u \in V_{1} \cup\left\{y_{1}, \ldots, y_{p}\right\}$, then $p<c\left(y_{j} u\right) \leq t$. Thus $|V(M)|-\left|V\left(G^{\prime}\right)\right| \geq$

$d\left(y_{j}\right)-(t-p)$. It follows that $2 t-p \geq \delta-(t-p)$. Hence $t \geq \frac{\delta+2 p}{3} \geq \frac{2\left\lceil\frac{2 \delta}{5}\right\rceil+\delta}{3} \geq\left\lfloor\frac{3 \delta}{5}\right\rfloor$, which is a contradiction. This completes the whole proof of Theorem 5 .

\section{Proof of Theorem 6}

Let $\delta=\delta(G)$. If $\delta \leq 3$, it is easy to check that our theorem holds. So now we assume that $\delta \geq 4$. Suppose our conclusion is not true. Let $M$ be a maximum rainbow matching of size $t$. Then $t \leq\left\lfloor\frac{2 \delta}{3}\right\rfloor-1$. Suppose that $E(M)=\left\{e_{1}, e_{2}, \ldots, e_{t}\right\}$ and $e_{i}=x_{i} y_{i}$. Moreover, without loss of generality, we assume that $c\left(e_{i}\right)=i$. Put $V_{1}=V-V(M)$. A color is called a new color if it is not in $c(M)$ and we call an edge uv special if $v \in V(M), u \in V_{1}$ and $c(u v)$ is a new color. For $v \in V(M)$, let $d_{s}(v)$ denote the number of the special edges incident with $v$. Let $V_{2}=\left\{v \mid v \in V(M), d_{s}(v) \geq 3\right\}$. We have the following claim.

Claim 1. For each edge $x_{i} y_{i} \in E(M)$, if $d_{s}\left(x_{i}\right)+d_{s}\left(y_{i}\right) \geq 3$, then either $d_{s}\left(x_{i}\right)=0$ or $d_{s}\left(y_{i}\right)=0$.

Proof. Otherwise, suppose that $d_{s}\left(x_{i}\right)+d_{s}\left(y_{i}\right) \geq 3$ and $d_{s}\left(x_{i}\right), d_{s}\left(y_{i}\right) \geq 1$. Then either $d_{s}\left(x_{i}\right) \geq 2$ or $d_{s}\left(y_{i}\right) \geq 2$. Assume that $d_{s}\left(x_{i}\right) \geq 2$. As $d_{s}\left(y_{i}\right) \geq 1$, we choose a special edge $y_{i} u$. By $d_{s}\left(x_{i}\right) \geq 2$, there is a special edge $x_{i} w$ such that $c\left(x_{i} w\right) \neq c\left(y_{i} u\right)$. Clearly, $u \neq w$, because $G$ is triangle-free. Now $M \cup\left\{x_{i} w, y_{i} u\right\} \backslash x_{i} y_{i}$ is a rainbow matching of size $t+1$, a contradiction.

Claim 2. $\left|V_{2}\right| \geq\left\lceil\frac{\delta}{3}\right\rceil$.

Proof. Let $x \in V_{1}$. If there is an edge $x y$ such that $c(x y) \notin c(M)$, then $y \in V(M)$. Otherwise, there is a rainbow matching $M \cup x y$ of size $t+1$, which is a contradiction. Let $E_{s}$ denote the set of all the special edges. Since each vertex in $V_{1}$ has degree at least $\delta$, $\left|E_{s}\right| \geq(\delta-t)\left|V_{1}\right| \geq\left(\left\lceil\frac{\delta}{3}\right\rceil+1\right)\left|V_{1}\right|$. Note that $\left|V_{1}\right|=|V(G)|-|V(M)| \geq 2 \delta-2\left(\left\lfloor\frac{2 \delta}{3}\right\rfloor-1\right) \geq$ $\frac{2 \delta}{3}+2 \geq 3$ (recall that if $G$ is triangle-free, then $|V(G)| \geq 2 \delta$ ). On the other hand, by Claim 1, for each edge $x_{i} y_{i} \in E(M)$, if $d_{s}\left(x_{i}\right)+d_{s}\left(y_{i}\right) \geq 3$, then $d_{s}\left(x_{i}\right)=0$ or $d_{s}\left(y_{i}\right)=0$. 
So $d_{s}\left(x_{i}\right)+d_{s}\left(y_{i}\right) \leq\left|V_{1}\right|$. Thus by Claim $1,\left|E_{s}\right| \leq\left|V_{2}\right|\left|V_{1}\right|+2\left(|E(M)|-\left|V_{2}\right|\right)$. So we have the following inequality: $\left(\left\lceil\frac{\delta}{3}\right\rceil+1\right)\left|V_{1}\right| \leq\left|V_{2}\right|\left|V_{1}\right|+2\left(|E(M)|-\left|V_{2}\right|\right)$. Hence

$$
\begin{aligned}
\left|V_{2}\right| \geq \frac{\left(\left\lceil\frac{\delta}{3}\right\rceil+1\right)\left|V_{1}\right|-2|E(M)|}{\left|V_{1}\right|-2} & \geq \frac{\left(\left\lceil\frac{\delta}{3}\right\rceil+1\right)\left|V_{1}\right|-2\left(\left\lfloor\frac{2 \delta}{3}\right\rfloor-1\right)}{\left|V_{1}\right|-2} \\
& =\left\lceil\frac{\delta}{3}\right\rceil+1-\frac{2\left\lfloor\frac{2 \delta}{3}\right\rfloor-2\left\lceil\frac{\delta}{3}\right\rceil-4}{\left|V_{1}\right|-2} \\
& \geq\left\lceil\frac{\delta}{3}\right\rceil .
\end{aligned}
$$

For each edge $e$ of $M$, at most one end vertex of $e$ is in $V_{2}$. Thus, without loss of generality, we assume that $V_{2}=\left\{x_{1}, x_{2}, \ldots, x_{p}\right\}$, where $p=\left|V_{2}\right| \geq\left\lceil\frac{\delta}{3}\right\rceil$. Let $G^{\prime}$ denote the subgraph induced by $\left\{y_{1}, y_{2}, \ldots, y_{p}\right\}$.

Claim 3. There is a vertex $v \in V_{2}$ such that $d_{s}(v) \geq 5$.

Proof. Otherwise, we have that each vertex $v \in V(M)$ has $d_{s}(v) \leq 4$. By Claim 1, it holds that for each edge $x_{i} y_{i} \in E(M), d_{s}\left(x_{i}\right)+d_{s}\left(y_{i}\right) \leq 4$. Then $\left|E_{s}\right| \leq 4\left(\left\lfloor\frac{2 \delta}{3}\right\rfloor-1\right)$. On the other hand, $\left|E_{s}\right| \geq\left|V_{1}\right|\left(\left\lceil\frac{\delta}{3}\right\rceil+1\right) \geq\left(\left\lceil\frac{2 \delta}{3}\right\rceil+2\right)\left(\left\lceil\frac{\delta}{3}\right\rceil+1\right)$. It follows that $4\left(\left\lfloor\frac{2 \delta}{3}\right\rfloor-1\right) \geq$ $\left(\left\lceil\frac{2 \delta}{3}\right\rceil+2\right)\left(\left\lceil\frac{\delta}{3}\right\rceil+1\right)$. Hence $2 \delta^{2}-12 \delta+54 \leq 0$, which is a contradiction.

Without loss of generality, we assume that $d_{s}\left(x_{1}\right) \geq 5$. By Claim $1, d_{s}\left(y_{1}\right)=0$.

Claim 4. If $y_{1}$ has a neighbor $y \in V\left(G^{\prime}\right) \cup V_{1}$, then $c\left(y_{1} y\right) \in c(M)$ and $c\left(y_{1} y\right) \notin$ $\{1,2, \ldots, p\}$.

Proof. We distinguish the following two cases:

Case 1. Assume that $y_{1}$ has a neighbor, say $y=y_{2} \in V\left(G^{\prime}\right)$. We prove it by contradiction. Firstly, suppose that $c\left(y_{1} y_{2}\right)$ is a new color. Then we can find two independent special edges $x_{1} w_{1}$ and $x_{2} w_{2}$ such that $c\left(x_{1} w_{1}\right), c\left(x_{2} w_{2}\right) \notin c(M) \cup\left\{c\left(y_{1} y_{2}\right\}\right.$ and $c\left(x_{1} w_{1}\right) \neq c\left(x_{2} w_{2}\right)$. We can do this, because $d_{s}\left(x_{1}\right) \geq 5$ and $d_{s}\left(x_{2}\right) \geq 3$. Now we obtain a rainbow matching $M \cup\left\{x_{1} w_{1}, x_{2} w_{2}, y_{1} y_{2}\right\} \backslash\left\{x_{1} y_{1}, x_{2} y_{2}\right\}$ of size $t+1$, which is a contradiction.

Next, suppose that $c\left(y_{1} y_{2}\right) \cap\{1,2, \ldots, p\} \neq \emptyset$. Since $G$ is properly colored, $c\left(y_{1} y_{2}\right) \neq$ 1,2 . Without loss of generality, we assume that $c\left(y_{1} y_{2}\right)=3$. As $d_{s}\left(x_{3}\right), d_{s}\left(x_{2}\right) \geq 3$ and $d_{s}\left(x_{1}\right) \geq 5$, we can easily find three special edges $x_{1} w_{1}, x_{2} w_{2}, x_{3} w_{3}$ to form a rainbow matching. Hence $M \cup\left\{x_{1} w_{1}, x_{2} w_{2}, x_{3} w_{3}, y_{1} y_{2}\right\} \backslash\left\{e_{1}, e_{2}, e_{3}\right\}$ is a rainbow matching of size $t+1$.

Case 2. $y_{1}$ has a neighbor $y \in V_{1}$. We prove it by contradiction. Firstly, suppose that $c\left(y_{1} y\right)$ is a new color. Then there is a special edges $x_{1} w_{1}$ such that $c\left(x_{1} w_{1}\right) \neq c\left(y_{1} y\right)$, because $d_{s}\left(x_{1}\right) \geq 5$. Now we obtain a rainbow matching $M \cup\left\{x_{1} w_{1}, y_{1} y\right\} \backslash\left\{x_{1} y_{1}\right\}$ of size $t+1$, which is a contradiction. 
Next, suppose that $c\left(y_{1} y\right) \cap\{1,2, \ldots, p\} \neq \emptyset$. Since $G$ is properly colored, $c\left(y_{1} y\right) \neq 1$. Without loss of generality, we assume that $c\left(y_{1} y\right)=2$. As $d_{s}\left(x_{2}\right) \geq 3$ and $d_{s}\left(x_{1}\right) \geq 5$, we can easily find two independent special edges $x_{1} w_{1}, x_{2} w_{2}$ such that $w_{2} \neq y$ to form a rainbow matching. Hence we can obtain a rainbow matching $M \cup\left\{x_{1} w_{1}, x_{2} w_{2}, y_{1} y\right\} \backslash\left\{e_{1}, e_{2}\right\}$ of size $t+1$. This contradiction completes the proof of Claim 4 .

Now consider the vertex $y_{1}$. By Claims 3,4 and $d_{s}\left(y_{1}\right)=0$, we know that if $y_{1}$ has a neighbor $u \in V_{1} \cup\left\{y_{1}, \ldots, y_{p}\right\}$, then $c\left(y_{1} u\right) \in c(M)$ and $c\left(y_{1} u\right) \notin\{1,2, \ldots, p\}$. Thus $\left|\left\{x_{1}, \ldots, x_{p}\right\}\right|+\left|\left\{e_{p+1}, \ldots, e_{t}\right\}\right| \geq d\left(y_{1}\right)-(t-p)$. It follows that $t \geq \delta-(t-p)$. Hence $t \geq \frac{\delta+p}{2} \geq \frac{\left\lceil\frac{\delta}{3}\right\rceil+\delta}{2} \geq\left\lfloor\frac{2 \delta}{3}\right\rfloor$, which is a contradiction. This completes the whole proof.

\section{Acknowledgement}

I would like to thank the referee for the careful review and the valuable comments. This research was supported by NSFC Grants $(61070230,11026184,10901097)$, IIFSDU (2009hw001), RFDP(20100131120017) and SRF for ROCS.

\section{References}

[1] B. Alspach, Problem 89, Discrete Math. 69 (1988) 106.

[2] R. P. Anstee and L. Caccetta, Orthogonal matchings, Discrete Math. 179 (1998) $37-47$.

[3] J. A. Bondy and U. S. R. Murty, Graph Theory with Applications, Macmillan Press, New York, 1976.

[4] R. A. Brualdi and H. J. Ryser, Combinatorial Matrix Theory, Cambridge University Press, Cambridge, UK, 1991.

[5] H. J. Ryser, Neuere probleme der kombinatorik, Vorträge über Kombinatorik Oberwolfach, Mathematisches Forschungsinstitut Oberwolfach, July 1967.

[6] T.D. LeSaulnier, C. Stocker, P.S. Wenger and D. B. West, Rainbow Matching in Edge-Colored Graphs, Electron. J. Combin. 17 (2010), \#N26.

[7] P. Hatami and P. W. Shor, A lower bound for the length of a partial transversal in a Latin square, J. Combin. Theory Ser. A 115 (2008), 1103-1113.

[8] M. Kano and X. Li, Monochromatic and heterochromatic subgraphs in edge-colored graphs - a survey, Graphs Combin. 24 (2008), 237-263.

[9] S. K. Stein, Transversals of Latin squares and their generalizations, Pacific J. Math. 59 (1975), 567-575.

[10] R. Stong, Orthogonal Matchings, Discrete Math. 256 (2002), 515-518.

[11] I. M. Wanless, Transversals in Latin squares: A survey, in R. Chapman (ed.), Surveys in Combinatorics 2011, London Math. Soc. Lecture Note Series 392, Cambridge University Press, 2011, pp403-437. 